\title{
Association between Serum IGF1 Levels and Liver fat content in patients with pituitary diseases.
}

Petit JM, Nguyen A, Ricolfi F, Aho LS, Bouillet B, Fourmont C, Loffroy R, Cercueil JP, Verges B Endocrinologie Diabétologie, Inserm U866, CHU Dijon France

Non-alcoholic fatty liver disease (NAFLD) is commonly associated with obesity, metabolic syndrome and type 2 diabetes. NAFLD is also seen in patients with endocrinopathies. However, the relationship between endocrinopathies and the development of NAFLD is not wel known.

It has been suggested that patients with hypothalamic and/or pituitary disease are at risk of excessive weight gain, impaired glucose tolerance, and dyslipidemia with subsequent development of NAFLD. In the same way, it seems that these patients have a high prevalence of cirrhosis placing them at risk for liver related death (Adams et al 2004). Both GH and IGF1 regulate hepatic lipid metabolism and could be involved in the pathophysiology of NAFLD in patients with pituitary diseases.

In this study, we set out to determine whether liver fat content (LFC) was associated with IGF1 levels and with GH deficiency in people with pituitary diseases (PD).

Design, Settings, and Participants- 88 patients with pituitary diseases and 74 healthy controls were included in this study. LFC was measured using MRI as previously described (Guiu et al 2009). Hepatic steatosis was defined as LFC $>5.5 \%$.

Serum IGF1 levels were analysed in the hospital laboratory using chemiluminescent immunometric assays (Siemens Medical Solutions Diagnostics, Erlangen, Germany) according to the manufacturer's instructions

RESULTS - Patients with PD were older $(p=0.001)$, had a higher BMI $(p=0.0005)$ and higher ALAT level $(p=0.0002)$ than healthy controls. LFC was significantly higher in people with PD than in controls $(6.5 \%$ vs $3.2 \%$; $p<0.001)$. LFC was negatively associated with the IGF1 level. Fourteen patients with PD had GH deficiency. LFC was higher in PD patients with GH deficiency than in those without. The prevalence of steatosis (LFC $>5.5 \%$ ) was higher in PD patients than in controls $(36.3 \%$ vs $14.8 \% ; p=0.002$ respectively). The prevalence of steatosis was higher in PD patients with GH deficiency than in those without $(12 / 14(85.7 \%)$ vs $20 / 74(27.0 \%) ; p<0.001)$.

In multivariate analysis, which included patients and controls, the predictive variables for steatosis were age, BMI and IGF1 levels, whereas the presence of pituitary diseases and gender were not associated with steatosis.

\begin{tabular}{|c|c|c|c|}
\hline & $\begin{array}{c}\text { Patients with } \\
\text { PD }\end{array}$ & Controls & p \\
\hline $\mathbf{n}$ & 88 & 74 & \\
\hline Age (years) & $54,4 \pm 14,7$ & $40,8 \pm 14,5$ & $\mathbf{0 , 0 0 2}$ \\
\hline BMI (kg/m2) & $29,5 \pm 6,6$ & $25,8 \pm 5,1$ & $<\mathbf{0 , 0 0 0 1}$ \\
\hline LDL (mmol/1) & $3,29 \pm 0,94$ & $3,09 \pm 0,75$ & 0,31 \\
\hline HDL (mmol/1) & $1,23 \pm 0,47$ & $1,36 \pm 0,39$ & 0,30 \\
\hline $\begin{array}{c}\text { Triglycérides } \\
\text { (mmol/1) }\end{array}$ & $1,90 \pm 1,6$ & $1,21 \pm 0,56$ & $\mathbf{0 , 0 0 9}$ \\
\hline $\begin{array}{c}\text { Glycémia } \\
\text { (mmol/1) }\end{array}$ & $5,52 \pm 0,9$ & $4,85 \pm 0,7$ & $\mathbf{0 , 0 0 1}$ \\
\hline $\begin{array}{c}\text { Liver fat content } \\
\text { (\%) }\end{array}$ & $6.5 \%$ & $3.2 \%$ & $<\mathbf{0 , 0 0 0 1}$ \\
\hline $\begin{array}{c}\text { steatosis (LFC } \\
>\mathbf{5 . 5 \% )}\end{array}$ & $32(36.3 \%)$ & $11(14.8 \%)$ & $\mathbf{0 . 0 0 2}$ \\
\hline
\end{tabular}

\begin{tabular}{|c|c|c|c|}
\hline & OR & $\mathrm{Cl}$ & $\mathbf{p}$ \\
\hline age & 1,003 & $1,001-1,077$ & 0,04 \\
\hline BMI & 1,33 & $1,18-1,49$ & $<0,001$ \\
\hline IGF1 & 0,99 & $0,98-0,99$ & 0,004 \\
\hline PD & 1,35 & $0,43-4,2$ & 0,59 \\
\hline gender & 0,57 & $0,21-1,49$ & 0,25 \\
\hline
\end{tabular}

\section{Characteristics of the Patient and controls groups}

DISCUSSION: - Our data showed that LFC was strongly associated with IGF1 levels. These results suggest that steatosis associated with pituitary diseases is probably a consequence of GH deficiency. The screening of NAFLD could be included in the follow-up of patients with pituitary diseases, and more specifically in patients with other risk factors of NAFLD such as obesity and metabolic syndrome. An evaluation of the interest of GH treatment in GH-deficient PD patients with NASH should be evaluated. 